\title{
Characterizing a Liquid Repellent Nano-Coating Using a Multi-Technique Approach
}

\author{
T. S. Nunney*, P. Mack*, O. Greenwood*, R. G. White*, M. H. Wall**, B. R. Strohmeier**, S. \\ Coulson***, D. Evans*** \\ * Thermo Fisher Scientific, The Birches, Imberhorne Lane, East Grinstead, West Sussex, RH19 \\ 1UB, UK. \\ ** Thermo Fisher Scientific, 5225 Verona Rd, Madison, WI 53711, USA. \\ *** P2i Limited, 127 North, Milton Park, Abingdon, Oxfordshire, OX14 4S, UK
}

Modern materials, such as fabrics, papers and polymers, have ideal bulk properties for applications which require flexibility, strength and low weight. It is often necessary, however, to modify the surface properties of the material to make it compatible with the environment in which it used, such as biological applications, or to make a particular fabric more oil or liquid repellent, without changing how it looks or feels.

A nano-coating has previously been developed (P2i Limited), which successfully improves the liquid repellent properties of a range of materials. The surface of poly(ethylene) terephthalate, for example, can be modified from slightly hydrophilic to significantly hydrophobic with this nano-coating. The coating is deposited using a novel plasma treatment, which aims to preserve the fluorine-containing monomer structure when the fluoropolymer structure is built on the surface. This gives the highest possible liquid repellency, whereas conventional plasma treatments break the monomer structure, degrading the potential repellent effect.

This presentation will show how the Thermo Scientific XPS systems, used for chemical analysis of the surface, and the Thermo Scientific DXR Raman microscope can be used to characterize these samples. The XPS system was used to softly profile the nano-coating on poly(ethylene) terephthalate and polytetrafluoroethylene substrates. A newly-developed argon cluster source was used for profiling, to minimize polymer damage during the analysis, and maintain the surface chemistry; a critical part of the analysis. The results are consistent with a preservation of the fluorine-containing monomer structure, as evinced by the film quantification and analysis of the $\mathrm{C} 1 \mathrm{~s}$ peaks shapes. These results are supplemented by Raman microscopy measurements, which will be discussed in terms of the molecular nature of the nanocoating. 